$(14 / 43)$ decreased their dose to $\leq 7.5 \mathrm{mg} /$ day by Year $10 ; 9.5 \%$ (9/95) of patients receiving baseline prednisone $\leq 7.5 \mathrm{mg} /$ day had a dose increase to $>7.5 \mathrm{mg} /$ day. Conclusions: Over 10 years $\mathrm{BEL}+\mathrm{SoC}$ was well tolerated and the rates and nature of AEs were consistent with the known profile of BEL. Efficacy was maintained and prednisone use decreased in those receiving $>7.5 \mathrm{mg} /$ day at baseline.

Acknowledgements: Study funded by GSK. Editorial assistance provided by Katie White, PhD, Fishawack Indicia Ltd, UK; funded by GSK.

Disclosure of Interest: D. Wallace Grant/research support from: GSK, Consultant for: GSK, E. Ginzler Grant/research support from: GSK, J. Merrill Grant/research support from: GSK, Bristol-Myers Squibb, Consultant for: Anthera Pharmaceuticals, Inc., GSK, EMD Serono, Inc., Lilly, AstraZeneca, Bristol-Myers Squibb, UCB, Celgene, Biogen, R. Furie Grant/research support from: GSK, Consultant for: GSK, W. Stohl Grant/research support from: GSK, Pfizer, Consultant for: Johnson \& Johnson, W. Chatham Grant/research support from: GSK, A. Weinstein Grant/research support from: GSK, HGS, Consultant for: GSK, HGS, J. McKay Grant/research support from: GSK, Medlmmune, Anthera Pharmaceuticals, Inc., Johnson \& Johnson, Lilly, Xencor, Inc., W. McCune Grant/research support from: GSK, Lilly, M. Petri Grant/research support from: GSK, Consultant for: GSK, J. Fettiplace Shareholder of: GSK, Employee of: GSK, D. Roth Shareholder of: GSK, Employee of: GSK, B. Ji Shareholder of: GSK, Employee of: GSK, A. Heath Shareholder of: GSK, Employee of: GSK

DOI: 10.1136/annrheumdis-2017-eular.6430

\section{OP0233 PRIMARY PROPHYLAXIS OF CARDIOVASCULAR EVENTS IN SYSTEMIC LUPUS ERYTHEMATOSUS: A RETROSPECTIVE ANALYSIS OF 291 PATIENTS FROM TWO ITALIAN CENTERS}

S. Fasano $^{1}$, D. Margiotta ${ }^{2}$, L. Navarini ${ }^{2}$, L. Pierro ${ }^{1}$, A. Riccardi ${ }^{1}$, I. Pantano ${ }^{1}$ A. Afeltra ${ }^{2}$, G. Valentini ${ }^{1} .{ }^{1}$ Rheumatology Unit, University of Campania "Luigi Vanvitelli", Naples; ${ }^{2}$ Unit of Allergology, Immunology, Rheumatology, Campus Bio-Medico University, Rome, Italy

Background: Systemic lupus erythematosus (SLE) is associated with an increased cardiovascular (CV) risk (1). By retrospectively investigating a one centre cohort, we have recently reported that low-dose aspirin (ASA) use is associated with a reduced CV risk in SLE (2) and long-term hydroxychloroquine (HCQ) exposure may have an additive effect (3).

Objectives: This study was conducted on 2 Italian SLE cohorts to confirm these results and assess the role, if any, of statins.

Methods: Clinical charts of SLE patients consecutively admitted to 2 University Rheumatology Units from November 2000 to December 2014 who, at admission, had not experienced any CV event, were investigated. ASA, HCQ and statins use and the occurrence of any CV event, were recorded at each visit. Kaplan-Meier analysis was performed to determine the HCQ exposure status associated with a higher CV-free survival. Cox regression analysis was carried out to identify factors independently associated with a first CV event.

Results: A total of 291 SLE patients were included in the study and followed for a median of 8 years. During follow-up, $16 \mathrm{CV}$ events occurred. Kaplan Meier analysis revealed a greater CV event-free rate in the 120 ASA-treated patients taking $\mathrm{HCQ}$ at standard dose for more than 5 years than in the 98 patients treated with ASA alone or with HCQ for less than 5 years (Figure 1). At univariate analysis, patients with a first $\mathrm{CV}$ event compared with those without any thrombotic events were antiphospholipid antibody (aPL) positive $(P=0.017$ HR 2.91) and had significantly higher blood pressure ( $P=0.017 \mathrm{HR} 3.58$ ), hypercholesterolemia ( $P=0,015$ HR 3.40) and higher disease damage at last visit ( $P=0,032$ HR 1.56). Moreover, ASA treatment ( $P=0,012 \mathrm{HR} 0.27)$ and HCQ use ( $P=0,012 \mathrm{HR} 0.26)$ for more than 5 years were negative predictors, while statins use did not show any association $(P=0.619)$. All other variables examined, including smoking, obesity, hypertriglyceridaemia, diabetes mellitus, disease activity, severe SLE,

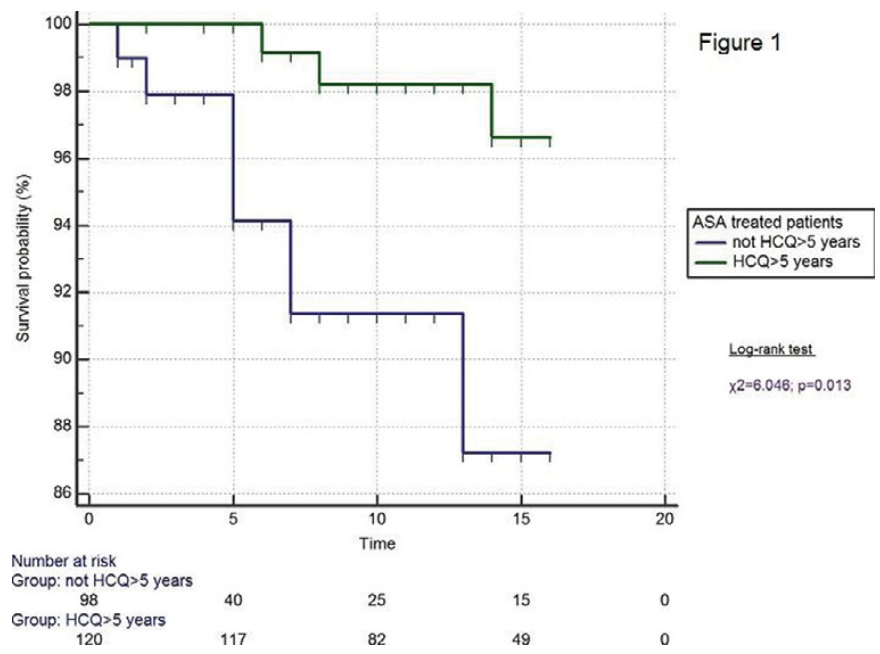

other medications (immunosuppressive agents, steroids) were not associated, either positively or negatively, with the occurrence of CV events. At multivariate analysis, taking ASA and HCQ for more than 5 years were protective against thrombosis (HR 0.24 and HR 0.27 , respectively), while aPL positivity (HR 4.32) increased the risk of a first CV event.

Conclusions: Use of antimalarials for more than 5 years is associated with a reduced risk of a first thrombosis in SLE patients and the HCQ-ASA combination seems to synergistically reduce further the CV risk. Larger, prospective studies are needed to provide a better definition of the role of these drugs in CV primary prevention in SLE.

References:

[1] Cervera R, et al. Morbidity and mortality in systemic lupus erythematosus during a 10-year period: a comparison of early and late manifestations in a cohort of 1,000 patients. Medicine (Baltimore). 2003.

[2] ludici $M$, et al. Low-dose aspirin as primary prophylaxis for cardiovascular events in systemic lupus erythematosus: a long-term retrospective cohort study. RheumatolOxfEngl 2016.

[3] Fasano S, et al. Long-Term Hydroxychloroquine Therapy and Low-Dose Aspirin May Have an Additive Effectiveness in the Primary Prevention of Cardiovascular Events in Patients with Systemic Lupus Erythematosus [abstract]. Arthritis Rheumatol 2016.

Disclosure of Interest: None declared

DOI: 10.1136/annrheumdis-2017-eular.2517

\section{OP0234 CLINICAL AND BIOLOGIC EFFECTS OF ICOSL BLOCKADE BY AMG 557 IN SUBJECTS WITH LUPUS ARTHRITIS}

L.E. Cheng ${ }^{1}$, Z. Amoura ${ }^{2}$, B. Cheah ${ }^{3}$, F. Hiepe ${ }^{4}$, B.A. Sullivan ${ }^{1}$, L. Zhou ${ }^{1}$, G.E. Arnold ${ }^{1}$, W.H. Tsuji ${ }^{1}$, J.T. Merrill ${ }^{5}$, J.B. Chung ${ }^{1} .{ }^{1}$ Amgen Inc., Thousand Oaks, United States; ${ }^{2}$ Hôpital Pitié-Salpêtrière, Paris, France; ${ }^{3}$ International Medical University, Kuala Lumpur, Malaysia; ${ }^{4}$ Charité Universitätsmedizin Berlin, Berlin, Germany; ${ }^{5}$ Oklahoma Medical Research Foundation, Oklahoma City, United States

Background: Blockade of inducible costimulatory ligand (ICOSL) might be a promising approach for autoimmune diseases such as systemic lupus erythematosus (SLE). We evaluated AMG 557, an anti-ICOSL monoclonal antibody, in an exploratory phase 1b study of SLE subjects with inflammatory arthritis, by withdrawing background therapies to improve interpretability of a small study. Objectives: To investigate potential efficacy, safety, and tolerability of AMG 557 in subjects with lupus arthritis.

Methods: This double-blind, randomized, placebo-controlled trial enrolled subjects with SLE and active lupus arthritis ( $\geq 4$ tender and 4 swollen joints) and Systemic Lupus Erythematosus Disease Activity Index [SLEDAI] score $>6$ despite stable background immunosuppressants. Upon enrollment, investigators were permitted to use up to $20 \mathrm{mg} /$ day of prednisone, which was tapered by day 85 to $7.5 \mathrm{mg} / \mathrm{day}$ or $50 \%$ of baseline, whichever was lower. Subjects received AMG $557210 \mathrm{mg}$ or placebo once weekly for 3 weeks followed by 10 additional doses every other week until day 155. At day 29, background immunosuppressants were withdrawn. The primary clinical endpoint was a composite Lupus Arthritis Responder Index at day 169; response was defined as achieving: (1) $50 \%$ decrease in combined tender and swollen joint counts, and (2) $\geq 1$ letter improvement in the musculoskeletal subsystem of the British Isles Lupus Assessment Group (BILAG) index and (3) prespecified immunosuppressant medication withdrawal and/or prednisone taper. Safety was a co-primary endpoint. Exploratory endpoints included safety, 4-point reduction of SLEDAI, clinical indices (SLEDAI, BILAG), complement components, autoantibodies, and lymphocyte populations.

Results: Twenty subjects (19 females) were randomized (10 AMG 557, 10 placebo) at 8 sites in North America, Asia, and Europe. The primary endpoint was met by $3 / 10$ subjects receiving AMG 557 and $1 / 10$ subjects receiving placebo $(P=$ ns). A 4-point decrease in SLEDAl score was achieved by $7 / 10$ subjects on AMG 557 and 2/10 subjects on placebo. At day 169, subjects receiving AMG 557 had decreases from baseline in mean global BILAG and SLEDAI scores of $36 \%$ and $48 \%$ respectively, compared to $25 \%$ and $11 \%$ in subjects receiving placebo. Lupus-associated biomarkers, including serum complement indices (C3, C4, and $\mathrm{CH} 50$ ), and autoantibodies, including anti-dsDNA, demonstrated trends towards improvement relative to placebo. Treatment-emergent adverse events (AEs) were similar between placebo and AMG 557 arms; most commonly headache and upper respiratory tract infection.

Conclusions: Results from this exploratory placebo-controlled trial in lupus arthritis suggest potential clinical benefit of ICOSL blockade by AMG 557 . These data support further clinical trials intervening in this costimulatory pathway in SLE and other autoimmune diseases.

Disclosure of Interest: L. Cheng Shareholder of: Amgen Inc., Employee of: Amgen Inc., Z. Amoura Consultant for: GlaxoSmithKline, AstaZeneca, BristolMyerSquibb, Amgen Inc., B. Cheah Grant/research support from: Merck, F. Hiepe Grant/research support from: Sanofi, Amgen Inc., GlaxoSmithKline, AstaZeneca, Ablynx, Consultant for: Baxalta, AstaZeneca, BristolMyerSquibb, GlaxoSmithKline, Novartix, Miltenyi, Neovacs, Hexal, Neovi, Speakers bureau: Pfizer Inc., Roche, UCB, B. Sullivan Shareholder of: Amgen Inc., Employee of: Amgen Inc., L. Zhou Shareholder of: Amgen Inc., Employee of: Amgen Inc., G. Arnold Shareholder of: Amgen Inc., Employee of: Amgen Inc., W. Tsuji Shareholder of: Amgen Inc., Johnson\&Johnson, Consultant for: Celimmune, Seattle Genetics, Resolve 
Therapeutics, Employee of: Formerly Amgen Inc.; Partner in the Cascadia Drug Development Group, J. Merrill Consultant for: EMD Serono, BristolMyerSquibb, Human Genome Sciences, UCB, AstraZeneca, Celgene, J. Chung Shareholder of: Amgen Inc., Employee of: Amgen Inc.

DOI: 10.1136/annrheumdis-2017-eular.1127

\section{OP0235 A NOVEL B CELL SPECIFIC IFN-I BIOMARKER IS ASSOCIATED WITH PLASMABLAST NUMBERS FOLLOWING B CELL DEPLETION THERAPY IN SLE}

Y.M. El-Sherbiny ${ }^{1,2}$, M.Y. Md Yusof ${ }^{1,2}$, E. Hensor ${ }^{1,2}$, A. Rawstron ${ }^{3}$, M. Wittmann ${ }^{1,2}$, P. Emery ${ }^{1,2}$, E.M. Vital ${ }^{1,2}{ }^{1}$ Leeds Institute of Rheumatic and Musculoskeletal Medicine, University of Leeds: ${ }^{2}$ National Institute of Health Research Leeds Musculoskeletal Biomedical Research Unit; ${ }^{3}$ Haematological Malignancy Diagnostic Service, Leeds Teaching Hospitals NHS Trust, Leeds, United Kingdom

Background: SLE is a Type I interferon (IFN-I) mediated disease with autoreactive B cells. Plasmablasts, the immediate progeny of B cells, are expanded in SLE and correlate with disease activity. We showed that their rate of regeneration after therapeutic B cell depletion with rituximab is variable and predicts relapse[1]. IFN-I has been shown in vitro to induce the differentiation of $B$ cells into plasmablasts. We previously showed that therapeutic B cell depletion with anti-CD20 mAb leads to a transient reduction in CD20-negative plasmablasts, following which plasmablasts repopulate and their numbers predict clinical relapse. We developed tetherin as a flow cytometric, cell-specific marker for IFN-I response.

Objectives: To test the hypothesis that memory B cell tetherin determines the rate of plasmablast repopulation after rituximab.

Methods: 117 rituximab-treated SLE patients were studied prosectively using BILAG-2004 and flow cytometry. In 97 responders we tested plasmablasts at 6 months as a predictor of clinical relapse before 12 months to validate our previous finding. In 50 patients pre-rituximab and 28 patients post-rituximab we performed additional flow cytometry to measure tetherin on each cell subset. Expression of 18 ISGs was measured using Taqman on PBMCs and an ISG score calculated. Results: We divided clinical responders to rituximab into earlier relapse $(12$ months) or later relapse (>12 months). As in our published discovery cohort, plasmablasts were strongly predictive of clinical relapse. ROC analysis indicated that a plasmablast count of $>0.0008 \times 10^{9} / \mathrm{L}$ at 6 months yielded $73 \%(95 \% \mathrm{Cl}$ $45-92 \%)$ sensitivity and $90 \%(95 \% \mathrm{Cl} 56-99 \%)$ specificity in predicting earlier relapse; area under the curve of 0.86

Plasmablast numbers after rituximab were associated with Memory $B$ cell tetherin $(\mathrm{R}=0.38, \mathrm{p}=0.047$ ) but not ISG score ( $\mathrm{R}=0.24, \mathrm{p}=0.219)$ (Table 1 ).

In Pre-rituximab there was no relationship between any IFN assay and plasmablast count. After rituximab treatment there was no correlation between plasmablast count and ISG expression, nor with monocyte or NK cell surface tetherin. However, memory B cell tetherin MFI was positively correlated with plasmablast count (Table 1).

Table 1

\begin{tabular}{lcc}
\hline Interferon assay & \multicolumn{2}{c}{ Plasmablast Count (cells $\left.\times 10^{9} / \mathrm{L}\right)$} \\
\cline { 2 - 3 } & Pre-rituximab $(n=50)$ & Post-rituximab $(n=28)$ \\
\hline ISG expression Score: & $-0.11, P=0.448$ & $0.24, P=0.219$ \\
Tetherin protein level: Monocytes & $-0.08, P=0.592$ & $0.20, P=0.296$ \\
T-cells & $-0.16, P=0.269$ & $0.32, P=0.096$ \\
NK cells & $-0.14, P=0.324$ & $0.05, P=0.795$ \\
Naïve B-cells & $-0.04, P=0.801$ & $0.30, P=0.121$ \\
Memory B-cells & $0.07, P=0.618$ & $0.38, P=0.047$ \\
\hline
\end{tabular}

Values are Spearman's Rank Correlation Coefficient and $P$ values.

Conclusions: Although interferon stimulated gene expression is commonly used to measure IFN-I activity, tetherin provides a cell-specific assay. We demonstrate that by measuring IFN-I response in B cells specifically, we could explain plasmablast differentiation, and thereby clinical outcome. Memory B cell tetherin is valuable to immunophetype SLE.

References:

[1] Vital et al. Arthritis Rheum. 2011 Oct;63(10):3038-47.

Disclosure of Interest: None declared

DOI: 10.1136/annrheumdis-2017-eular.6423

\section{OP0236 BENEFIT AND SAFETY OF ANTITHROMBOTIC TREATMENT IN 264 PREGNANCIES IN PATIENTS WITH ANTIPHOSPHOLIPID SYNDROME}

C.M. Yelnik ${ }^{1}$, M. Lambert ${ }^{1}$, E. Drumez ${ }^{2}$, V. Le Guern ${ }^{3}$, J.-L. Bacri ${ }^{4}$, M. Guerra ${ }^{5}$, C.A. Laskin ${ }^{6}$, W. Branch ${ }^{7}$, L.R. Sammaritano ${ }^{5}$, N. Morel ${ }^{3}$, G. Guettrot-Imbert $^{3}$, D. Launay ${ }^{1}$, E. Hachulla ${ }^{1}$, P.-Y. Hatron ${ }^{1}$, J.E. Salmon ${ }^{5}$,

N. Costedoat-Chalumeau ${ }^{3} .{ }^{1}$ Internal Medicine Department, University of Lille, UFR Medicine, Universitary Hospital Center of Lille; ${ }^{2}$ Biostatistics Department, University of Lille, EA 2694. Universitary Hospital Center of Lille, lille: ${ }^{3}$ Internal Medicine Department, Paris Descartes-Sorbonne Paris Cité University, INSERM U1153, Cochin Hospital, Paris; ${ }^{4}$ Internal Medicine Department, Hospital Center of Valenciennes, Valenciennes, France ${ }^{5}$ Rheumatology, Hospital for Special
Surgery, New York City, United States; ${ }^{6}$ University of Toronto and LifeQuest Center for Reproductive Medicine, Toronto, Canada; ${ }^{7}$ University of Utah and Intermountain Healthcare, Salt Lake City, United States

Background: The management of pregnancy in patients with antiphospholipid syndrome (APS) with aspirin and heparin is based on empiric recommendations. Objectives: Our study aimed to evaluate the outcomes of treated patients with thrombotic and obstetric APS and the safety of antithrombotic treatments prescribed during pregnancy.

Methods: Inclusion criteria were (1) APS (Sydney criteria), (2) live pregnancy at 12 weeks of gestation (WG) with (3) follow up data until 6 weeks post-partum. Data were collected prospectively (PROMISSE study) and retrospectively (four French centers). Adverse pregnancy outcomes (APOs) were defined by fetal death or neonatal death; pre-term delivery before 36 WG due to preeclampsia or placental insufficiency; or small for gestational-age (SGA; $<5$ th percentile). Major bleeding was defined as blood loss greater than $500 \mathrm{~mL}$ and/or requiring surgery or transfusion.

Results: 264 pregnancies (87 collected prospectively) in 204 patients were included ( $46 \%$ with a history of thrombosis, and $23 \%$ with associated systemic lupus erythematosus). During pregnancy, treatment included heparin $(n=253$; $96 \%)$ and low-dose aspirin ( $n=223 ; 84 \%)$.

The live birth rate was $86 \%$. APOs occurred in $32 \%$, mostly during the 2nd trimester: fetal deaths $11 \%$, SGA $11 \%$, pre-term delivery before 36 WG due to preeclampsia or placental insufficiency $17 \%$. Thirteen maternal thrombotic events occurred in $12(4.5 \%)$ pregnancies. Forty-six maternal hemorrhagic events occurred in $40(15 \%)$ pregnancies (30 events in the post-partum period). Major bleeding was reported in only 6 pregnancies $(2.3 \%)$ and occurred only after delivery. Except for two events, post-partum hemorrhage occurred in the early post-partum before hospital discharge. No maternal death was observed.

Aspirin therapy during pregnancy was the only independent factor associated with a lower risk of APOs (odds ratio: $0.34 ; 95 \% \mathrm{Cl}: 0.15-0.78 ; \mathrm{p}=0.01$ ) in multivariate analysis.

Neither heparin or aspirin alone, nor combined therapy increased the risk of hemorrhage. In the retrospective cohort, emergency caesarian section was the only factor associated with hemorrhagic events during the study period $(53 \%$ hemorrhages in patients who underwent emergency caesarian compared to $18 \%$, $\mathrm{p}=0.005$ ). Independent risk factors for APOs were elevated body mass index and the presence of lupus anticoagulant.

Conclusions: We report a high level of obstetrical complications in conventionallytreated APS pregnancies, and a beneficial effect of addition of aspirin to prevent obstetrical morbidity. Moreover, heparin and aspirin were well tolerated and did not increase risk of hemorrhage.

Disclosure of Interest: None declared

DOI: 10.1136/annrheumdis-2017-eular.1926

\section{OP0237 LIFITEGRAST OPHTHALMIC SOLUTION 5.0\% FOR TREATMENT OF DRY EYE DISEASE: COMBINED EVIDENCE FROM 5 RANDOMIZED CONTROLLED TRIALS}

C. Baudouin ${ }^{1}$, M. Darvish-Zargar ${ }^{2}$, E.J. Holland ${ }^{3}$, C.C. Chan ${ }^{4}$, K.K. Nichols ${ }^{5}$, J. Tauber ${ }^{6}$, A. Raychaudhuri ${ }^{7}$, M. Roy ${ }^{7}$, A. Shojaei ${ }^{7}{ }^{1}$ Centre Hospitalier National d'Ophtalmologie des Quinze-Vingts, Paris, France; ${ }^{2}$ McGill University, Montreal, Canada; ${ }^{3}$ Cincinnati Eye Institute, Edgewood, United States;

${ }^{4}$ University of Toronto, Toronto, Canada; ${ }^{5}$ University of Alabama School of Optometry, Birmingham; ${ }^{6}$ Tauber Eye Center, Kansas City; ${ }^{7}$ Shire, Lexington, United States

Background: Dry eye disease (DED) is a multifactorial disease of the tear film and ocular surface, characterized by ocular discomfort and visual disturbance. DED is associated with a number of systemic autoimmune diseases, particularly rheumatoid arthritis and Sjögren's syndrome. ${ }^{2,3}$ Lifitegrast is a lymphocyte function-associated antigen-1 (LFA-1) antagonist that inhibits T-cell-mediated inflammation (an underlying factor in DED) and is approved in the US for the treatment of signs and symptoms of DED (lifitegrast ophthalmic solution $5.0 \%$, Xiidra ${ }^{\circledR}$.

Objectives: To evaluate the combined evidence from 5 clinical trials of lifitegrast ophthalmic solution $5.0 \%$ (LIF) in subjects with dry eye disease (DED).

Methods: Adults with DED were randomized to LIF or placebo (PBO) in 5 randomized, double-masked, placebo-controlled trials: 4 12-week efficacy/safety studies (phase 2, LIF $n=58$, PBO $n=58$; phase 3 trials: OPUS-1, LIF $n=293$, PBO $n=295$; OPUS-2, LIF $n=358$, PBO $n=360 ;$ OPUS-3, LIF $n=355$, PBO $\mathrm{n}=356$ ), and a 1-year safety study (SONATA, LIF $\mathrm{n}=220, \mathrm{PBO} n=111$ ). Individuals with secondary Sjögren's syndrome associated with autoimmune disease (eg, rheumatoid arthritis, systemic lupus erythematosus) were eligible to participate if they were not immunodeficient/immunosuppressed, not taking steroids, and met all other inclusion and exclusion criteria. Change from baseline to day 84 in DED signs and symptoms was evaluated across the 12-week studies. Key measures were inferior corneal staining score (ICSS; 0-4 scale), eye dryness score (EDS; visual analogue scale [VAS], 0-100 scale), and visual-related function subscale of a symptom scale ( $0-4$ scale). Pooled safety data (LIF $n=1287$, PBO $n=1177$ ) from all 5 trials were also analyzed.

Results: LIF improved ICSS versus PBO in the phase 2 study (secondary endpoint; treatment effect 0.35 , nominal $P=0.0209$ ), OPUS-1 (co-primary; 0.24, 